\title{
Improving the Shielding Effectiveness of a Board-Level Shield by Bonding it with the Waveguide-Below-Cutoff Principle
}

\author{
Andy Degraeve, Davy Pissoort \\ KU Leuven, Technology Campus Ostend \\ Research Group ReMI \\ Reliability in Mechatronics \& ICT \\ Zeedijk 101, Ostend, Belgium \\ andy.degraeve@kuleuven.be \\ davy.pissoort@kuleuven.be
}

\author{
Keith Armstrong \\ Cherry Clough Consultants Ltd \\ 9 Bracken View \\ Brocton \\ Stafford \\ Staffs, United Kingdom \\ keith.armstrong@cherryclough.com
}

\begin{abstract}
This paper discusses the shielding performance or shielding effectiveness of a board-level shield in function of its bonding method. Improved shielding performance at board-level in order to harden integrated circuits against unintentional and intentional electromagnetic interference, and this under harsh environmental conditions, is getting more and more important to achieve the desired levels of functional performance and operational reliability despite an ever more aggressive electromagnetic environment. High levels of operational reliability are increasingly being required to help control functional safety or other risks. As a board-level shield on its own only provides 5 of the 6 required walls to form a complete Faraday Cage, its overall shielding performance depends heavily on the way it is bonded to the printed circuit board's ground plane. It is shown by fullwave simulations that the shielding effectiveness can improve by more than $40 \mathrm{~dB}$ when bonded with the waveguide-below-cutoff principle compared to a classic perimeter bond of a single row of vias. And this even if the waveguides-below-cutoff are formed by rows of vias. Finally, the paper stresses the influence that internal resonances of the board-level shield have on its shielding effectiveness.
\end{abstract}

\section{INTRODUCTION}

The importance and need for Board-Level Shielding (BLS) on PCB motherboards, PCBs for mobile applications, etc. is continuously increasing because of several reasons. First, Integrated Circuits (ICs) are increasingly susceptible to electromagnetic interference because of die shrinking and lower internal voltages and noise margins [1]. Second, the electromagnetic environment is getting more polluted with electromagnetic disturbances of diverse nature and covering a wider frequency range. Third, on-board interference is getting more and more an issue because of tighter integration and inclusion of e.g. on-board wireless transmitters [2], [3].

The two weakest links are the bonding and conductors penetrating the shield. This paper focuses on the first phenomenon. The second one could be solved by appropriate filtering or using optical signals. Understanding of how to achieve a higher level of shielding effectiveness (SE) and a better understanding of the impact of different bonding strategies becomes stringent. Improved SE at board-level over a wide frequency range can harden ICs against unintentional and intentional electromagnetic interference and thus under harsh environmental conditions, thereby contributing to the increased levels of functional performance and operational reliability.

This paper studies the SE of a BLS in function of the way it is bonded to the ground plane. More specifically, this paper applies the waveguide-below-cutoff principle to achieve higher levels of SE than possible with the "classic" perimeter bond consisting of a single row of vias connecting the BLS to the PCB's ground plane. Both ideal, solid waveguides and a practical realisation based on parallel rows of vias are considered.

It is shown by means of full-wave simulations that the SE improves significantly by integrating the waveguide-belowcutoff principle. However, internal resonances can reduce the $\mathrm{SE}$ of the BLS, even below the cutoff frequencies of the applied waveguides. It is shown that these resonance frequencies depend not only on the BLS' dimensions, but also on what is inside the BLS.

This paper is organised as follows. Section II describes in general the waveguide-below-cutoff technique. Section III applies this technique to a BLS. Section IV describes the shielding performance degradations due to resonances as a consequence of physical dimensions of the BLS and its internal. Finally, Section V draws concluding remarks.

\section{WAVEguide(s)-Below-CutofF}

Although waveguides are usually applied to guide waves or signals with losses that are as low as possible, the physical mechanisms behind waveguides also allow to exploit them to block waves/signals.

Figure 1 depicts a rectangular waveguide with side dimensions $a$ and $b$ in the direction of the $x$ - and $y$-axis, respectively. The waveguide is assumed to be uniform along the $z$-axis. The waveguide's medium is assumed to be lossless $(\sigma=0)$ 


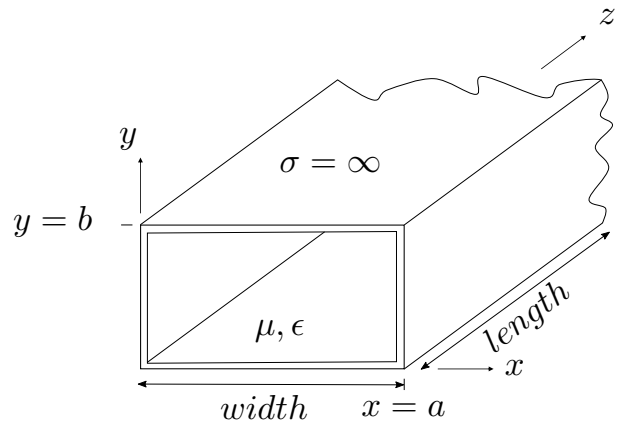

Fig. 1. A rectangular waveguide with side dimensions $a$ and $b$ in the direction of the $x$ - and $y$-axis, respectively. The waveguide is assumed to be uniform along its $z$-axis. The waveguide's medium is to be assumed lossless $(\sigma=0)$ and characterised by its permittivity $\epsilon$ and permeability $\mu$.

and characterised by its permittivity $\epsilon$ and permeability $\mu$. The $x$-axis is assumed to coincide with the larger transverse dimension by convention. Further, it is assumed that the walls are constructed form a perfect conductor $(\sigma=\infty)$.

As is well-known, a rectangular waveguide can only support $T E_{m n}$ and $T M_{m n}$ modes in contrast to e.g. a coaxial cable which also supports a $T E M$ mode. A $T E_{m n}$ mode refers to a field configuration where the electrical field is transverse to the direction of propagation ( $z$-axis in this case), thus $E_{z}=0$. A $T M_{m n}$ mode refers to a field configuration where the magnetic field is transverse to the direction of propagation, thus $H_{z}=0$. Here, the subscripts $m$ and $n$ indicate the order of the mode or in other words the number of half-wavelengths of the wave fitting along the $x$ - and $y$-axis, respectively.

An important property of a $T E_{m n}$ or $T M_{m n}$ mode is that it is not propagating along the $z$-axis from DC, but only from a given frequency called the cutoff frequency $f_{c, m n}$ of that mode. This in contrast to a $T E M$ mode which is propagating from DC. The cutoff frequency of $T E_{m n}$ and $T M_{m n}$ modes can be easily derived based on solving Maxwell's equations for the sourceless waveguide geometry [4]:

$$
f_{c, m n}^{T E, T M}=\frac{1}{2 \pi \sqrt{\mu \epsilon}} \sqrt{\left(\frac{m \pi}{a}\right)^{2}+\left(\frac{n \pi}{b}\right)^{2}}
$$

Here $m$ and $n$ are both integer numbers $(0,1,2,3, .$.$) which$ cannot be zero simultaneously.

Below the cutoff frequency, all waves are attenuated along the $z$-axis with an attenuation factor $\alpha_{m n}^{T E, T M}$ that is given by [4]:

$$
\alpha_{m n}^{T E, T M}=\omega \sqrt{\mu \epsilon} \sqrt{\left(\frac{f_{c, m n}}{f}\right)^{2}-1} \quad f \leq f_{c, m n}
$$

Exploiting this attenuation to block or shield signals below the lowest cutoff frequency is referred to as the waveguidebelow-cutoff principle.

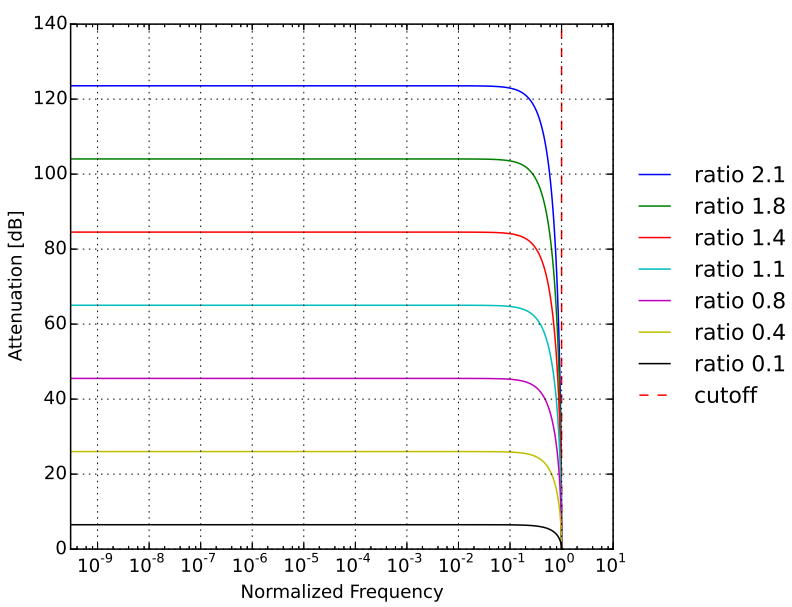

Fig. 2. Attenuation of a single rectangular waveguide in function its ratio defined as length/width. The frequency is normalized to the cutoff frequency.

In that case, the attenuation constant and the waveguide length $l$ contribute to the overall SE:

$$
S E_{d B}=20 \log _{10}\left(e^{\alpha_{m n} l}\right)
$$

Values for a single rectangular waveguide in function of a given length to aperture ratio are shown in Figure 2. The overall SE will be reduced in case of multiple waveguides [5], [6].

\section{WAVEguide(s)-Below-CutofF Applied to BLS}

In this section the simulation methodology to determine the SE of a BLS is described. Radiated or leaking field values are quantified by means of full-wave simulations and afterwards post-processed to obtain the SE. All full-wave simulations below are done with the CUDA enabled Finite-Difference Time-Domain (FDTD) solver that is included in Keysight Technologies' 3D Electromagnetic Simulation Platform EMPro [7].

\section{A. Shielding Effectiveness}

As motivated in [8]-[11], the shielding performance of a BLS should be estimated based on an average SE which is defined as the ratio of the total radiated power in the unshielded case to the total radiated power in the shielded case [12], [13]:

$$
S E_{d B}=10 \log _{10}\left(\frac{\left|P_{\text {rad,unshielded }}\right|}{\left|P_{\text {rad,shielded }}\right|}\right)
$$

In the simulations, the total radiated power is captured for both the unshielded set-up and the shielded set-up by a spherical far-zone sensor. 


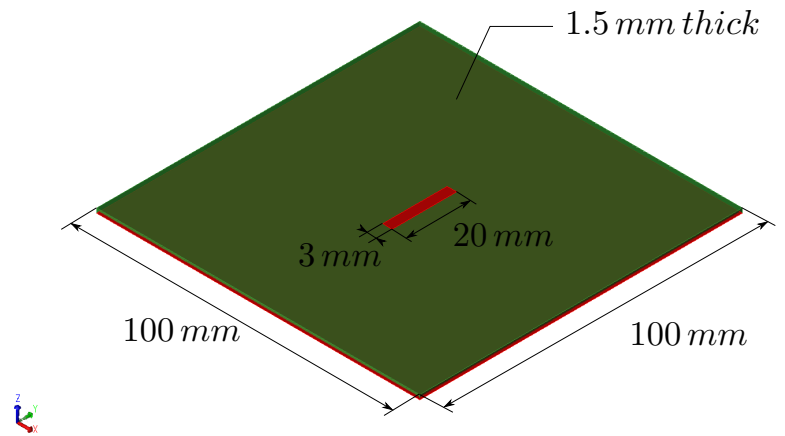

Fig. 3. Reference simulation model for the unshielded set-up. The PCB is a square double-layer PCB. The bottom layer of the PCB is a solid ground plane. The top layer contains a microstrip terminated in $50 \Omega$ and excited by a 1 Volt $50 \Omega$ broadband wave source.

\section{B. Classic Bonding}

The most common BLS shielding technique is to attach the BLS to the PCB by connecting it to the PCB's ground plane by a single row of vias [6]. This is referred to as the "classic" bonding.

1) Simulation Model: Figure 3 shows the simulation model for the unshielded set-up. The PCB is a square double-layer PCB with edge length of $100 \mathrm{~mm}$, thickness of $1.5 \mathrm{~mm}$, and a relative permittivity $\epsilon_{r}$ of 4.6 . The bottom layer of the PCB is a solid ground plane. The top layer of the PCB contains a microstrip with dimensions of 20 by $3 \mathrm{~mm}$ whose centre coincides with the PCB's centre. The microstrip is terminated in $50 \Omega$ and excited by a 1 Volt $50 \Omega$ broadband wave source.

Figure 4 shows the simulation model for the shielded setup. The BLS is continuously-bonded along its perimeter by equidistant vias. In the figure, the BLS is bonded by nine vias per side in which case they are $4.5 \mathrm{~mm}$ spaced. The BLS outer dimensions measures 40.5 by 40.5 by $10.5 \mathrm{~mm}$ with a wall thickness of $0.5 \mathrm{~mm}$. A guard ring is added to the setup, to bring the ground plane to the top layer of the PCB, connecting the perimeter of the BLS with the aid of vias to the ground plane. This single ring of vias is referred to as the "classic bonding technique" throughout this paper. The guard ring width is kept at $10 \mathrm{~mm}$ throughout all simulation setups. Future work will investigate the effect of changing its width. Simulations are set up for zero, two, three, five and nine vias to study the influence of only the bonding technique on the overall SE. The FDTD mesh cell's dimensions are kept constant to ensure the comparability and consistency of the results.

2) Results: Figure 5 shows the SE for the shielded set-up by applying the "classic bonding technique". It can be observed that: (i) the SE increases with the number of vias per side, and (ii) the shielding performance is degraded for frequencies above $1.5-2 \mathrm{GHz}$ depending on the distance between the vias.

Although both the SE as the maximal frequency could be improved by adding more and more vias per side, the PCB

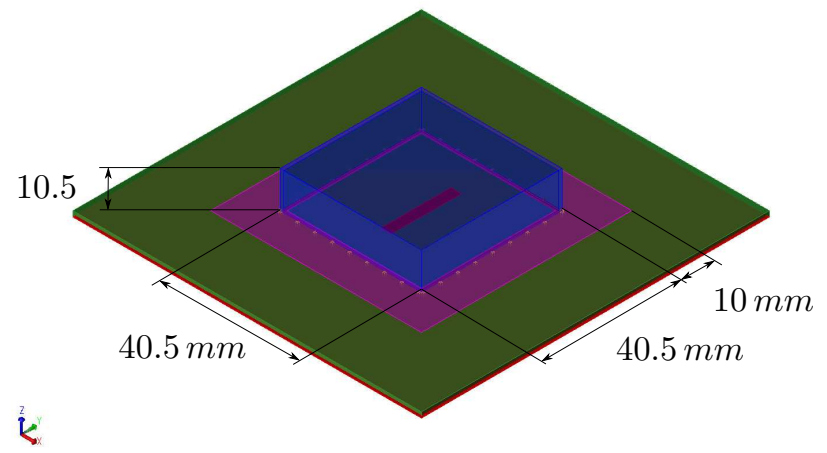

Fig. 4. Simulation model for the shielded set-up. The BLS is bonded along its perimeter by equidistant vias. In the figure, the BLS is bonded by nine vias per side in which case they are $4.5 \mathrm{~mm}$ spaced.

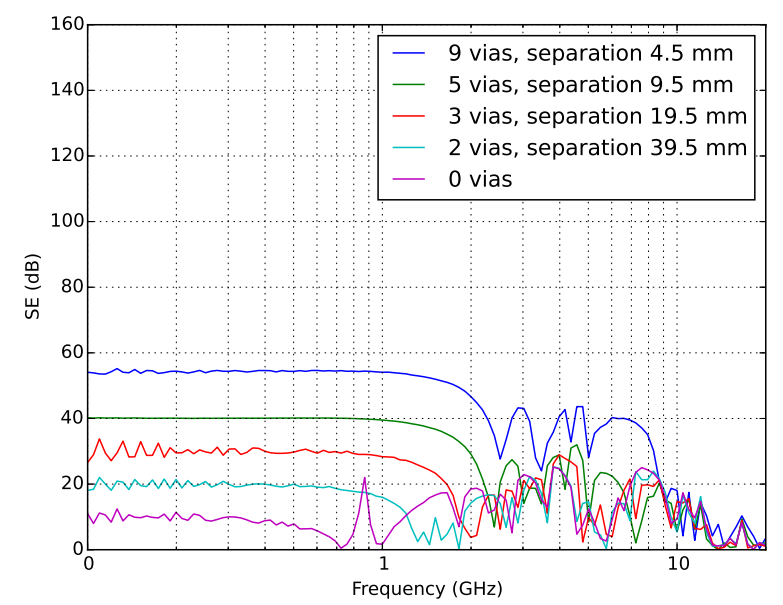

Fig. 5. Shielding effectiveness in function of frequency for the shielded setup by applying the "classic bonding technique". It can be observed that: (i) the SE increases with the number of vias per side, and (ii) the shielding performance is degraded for frequencies above $1.5-2 \mathrm{GHz}$ depending on the distance between the vias.

manufacturing process as well as the final mechanical stability of the PCB put a practical limit to this. Therefore, the question arises if there are other ways of bonding to increase the SE of the BLS.

\section{Ideal "Solid" Waveguides}

1) Simulation Model: Figure 6 shows the simulation model for a second shielded set-up. The BLS is now bonded by "solid" waveguides to the ground plane. "Solid" refers here to the equidistant parallel solid walls. The walls are $4.5 \mathrm{~mm}$ distant and have a thickness of $0.5 \mathrm{~mm}$. The PCB, BLS and microstrip have the same dimensions and properties as described before. The "Solid" waveguides are formed in the PCB's substrate by: (i) the guard ring, together with (ii) the equidistant parallel solid walls, and (iii) the ground plane. The waveguides have a cutoff frequency of $33.31 \mathrm{GHz}$.

2) Results: Figure 7 shows the SE for the "solid" waveguide bonding technique in function of different waveguide 


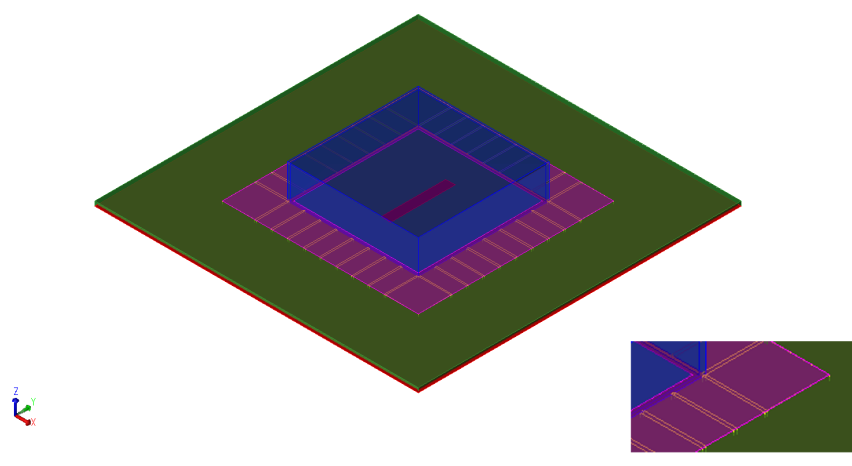

Fig. 6. Simulation model for a second shielded set-up. The BLS is now bonded by "solid" waveguides to the ground plane. "Solid" refers here to the equidistant parallel solid walls. The PCB, BLS and microstrip have the exact same dimensions and properties as described before. "Solid" waveguides are formed in the PCB's substrate. The waveguides have a cutoff frequency of $33.31 \mathrm{GHz}$

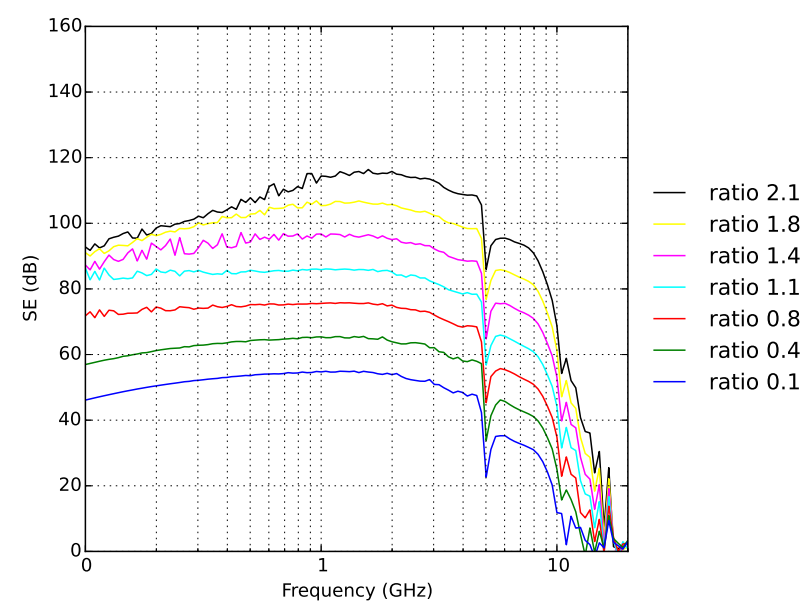

Fig. 7. Shielding effectiveness for the "solid" waveguide bonding technique in function of different waveguide ratios. It can be observed that by increasing the waveguide's ratio, the SE also increases as is expected from Equation (3).

ratios. It can be observed that: (i) the $\mathrm{SE}$ increases with the ratio, and (ii) the shielding performance is degraded for frequencies above $5 \mathrm{GHz}$. There is an improvement in: (i) the $\mathrm{SE}$, and (ii) the frequency of which the shielding performance starts to degrade compared to the "classic bonding technique". By increasing the ratio, the SE also increases as is expected from Equation (3) due to the increase of attenuation of frequencies below the cutoff frequency. The SE increases at the low frequency end for increasing frequencies due to nearfield magnetic shielding properties of the BLS.

\section{Practical "Via Waveguides"}

Solid parallel walls are, however, not incorporable into modern PCB design. This section models the waveguides by replacing the solid walls with parallel rows of vias.

1) Simulation Model: Figure 8 shows the simulation model for a third shielded set-up. The BLS is now bonded by "via

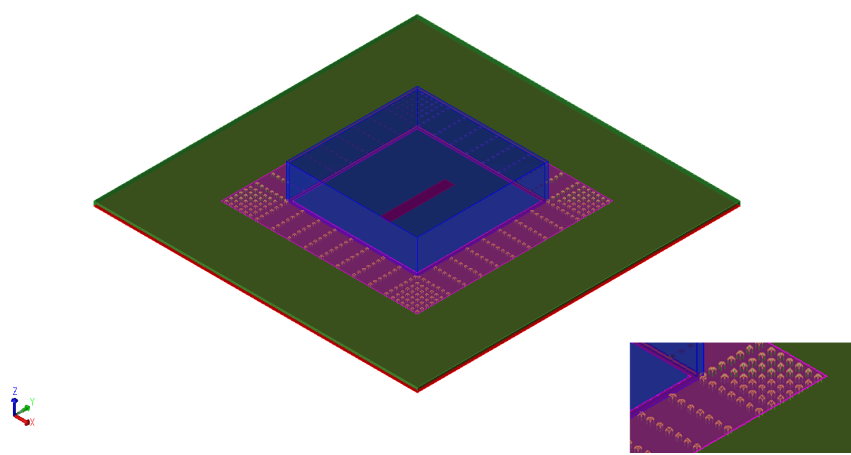

Fig. 8. Simulation model for a third shielded set-up. The BLS is now bonded by "via waveguides". "Via waveguides" refers to the construction of non-solid walls by the aid of $1 \mathrm{~mm}$ spaced consecutive vias. Each via added to lengthen the "via wall" results in an additional concentric "ring", thus a wall consisting of one via give rise to one concentric ring, a wall consisting of two consecutive vias give rise to two concentric rings and so on. The PCB, BLS and microstrip have the same dimensions and properties as described before.

waveguides". "Via waveguides" refers to the construction of non-solid walls by the aid of $1 \mathrm{~mm}$ spaced consecutive vias. Each via added to lengthen the "via wall" results in an additional concentric "ring". The PCB, BLS and microstrip have the same dimensions and properties as described before.

2) Results: Figure 9 shows the SE for the practical "via waveguide" bonding technique in function of the number of rings formed by the lengthwise consecutive spaced vias. It can be observed: (i) the SE increases with the number of rings, (ii) the shielding performance is degraded for frequencies above 3 - $5 \mathrm{GHz}$ depending on the number of rings. The one ring is not really considered in the previous range as it is close to the result of the "classic bonding technique". By increasing the number of via rings, the length of the waveguides increases, hence the ratio increases, thus the SE. The SE increases at the low frequency end for increasing frequencies due to nearfield magnetic shielding properties of the BLS. Overall, the SE achieved by the "via waveguide" bonding technique are quite close to the ones of the "solid" waveguides.

\section{RESONANCES}

Based on the previous figures, one sees that the SE of a BLS decreases in quite narrow bands even below the cutoff frequencies of the applied waveguides. As will be shown below, these can be related to cavity and structural resonances which reduce the overall SE. Empty cavity resonances can be approximated by the aid of closed-form formulas. More complex cases have to be numerically calculated with the aid of an eigenmode solver. All eigenmode calculations below are done with the Finite Element Method (FEM) Eigenmode solver that included in Keysight Technologies' 3D Electromagnetic Simulation Platform EMPro.

\section{A. Closed-form calculations}

The resonances occurring from the dimensional properties of the cavity can be calculated by [14]: 


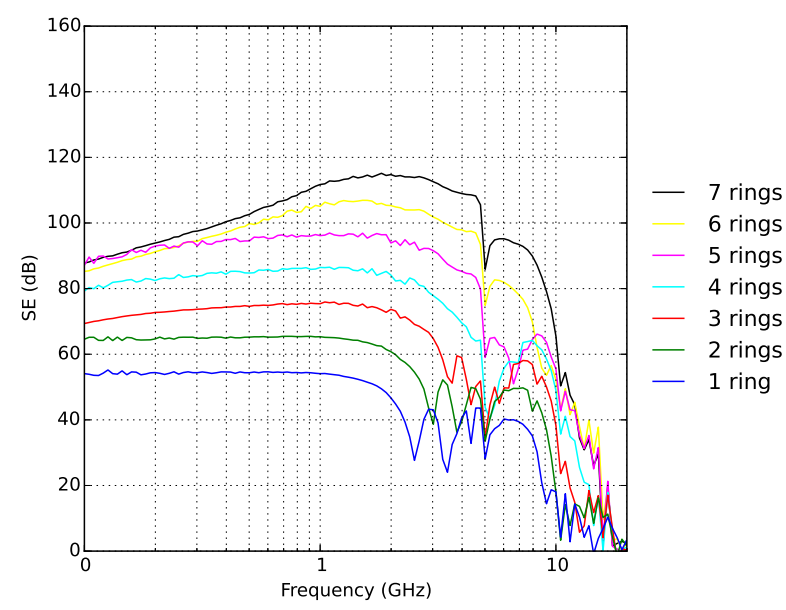

Fig. 9. for the practical "via waveguide" bonding technique in function of the number of rings formed by the lengthwise consecutive spaced vias. It can be observed: (i) the SE increases with the number of rings, (ii) the shielding performance is degraded for frequencies above $3-5 \mathrm{GHz}$ depending on the number of rings.

$$
f_{\text {res }}=\frac{1}{2 \pi \sqrt{(\mu \epsilon)}} \sqrt{\left(\frac{m \pi}{a}\right)^{2}+\left(\frac{n \pi}{b}\right)^{2}+\left(\frac{p \pi}{c}\right)^{2}}
$$

Here, $\mu$ and $\epsilon$ are respectively the permeability and the permittivity of the medium. $m, n$ and $p$ should be positive integers but no more than one can be zero at the same time. $a$ is the length, $b$ is the width and $c$ is the height of cavity in meters. The formula is only valid for homogeneously filled cavities.

\section{B. Simulation Model (FEM eigenmode solver)}

Figure 10 shows the simulation model for the determination of the eigen- or resonance frequencies. The idea is to model only the inside of the BLS. The perfect electric conducting sides of the BLS are achieved by applying this property to the boundary conditions of the FEM simulation domain. The block on top of the PCB, microstrip and guard ring represents free space.

Four set-ups are studied. In the first set-up the PCB, microstrip and guard ring are left out yielding a free space block matching the inner dimensions of the BLS. The second set-up adds the PCB. The third set-up adds the microstrip. The fourth set-up adds the guard ring.

\section{Results}

Figure 11 shows the resonance frequencies in function of the structural elements. It can be observed that there is a good agreement between the analytical approximation and numerical calculation for the first set-up. Obviously a sufficient number of modes should be calculated.

Adding the PCB shifts the resonance frequencies slightly down - e.g. the resonance frequency of $5.37 \mathrm{GHz}$ moves to $5.07 \mathrm{GHz}$ - and introduces new resonances in the upper

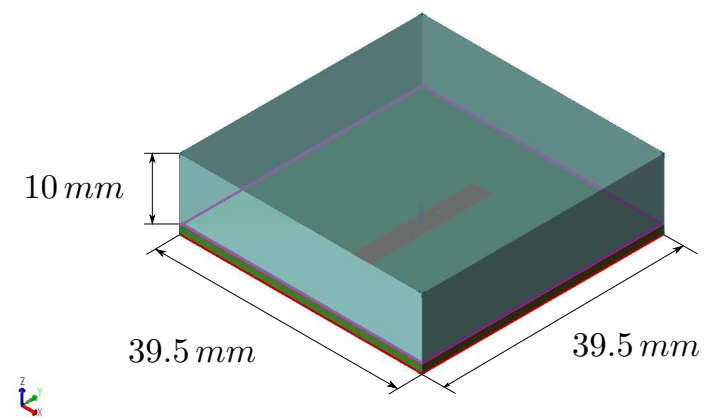

Fig. 10. Simulation model for the determination of the eigenfrequencies. The idea is to model only the inside of the BLS. The perfect electric conducting sides of the BLS are achieved by applying this property to the boundary conditions of the FEM simulation domain. The block on top of the PCB and microstrip represents free space.

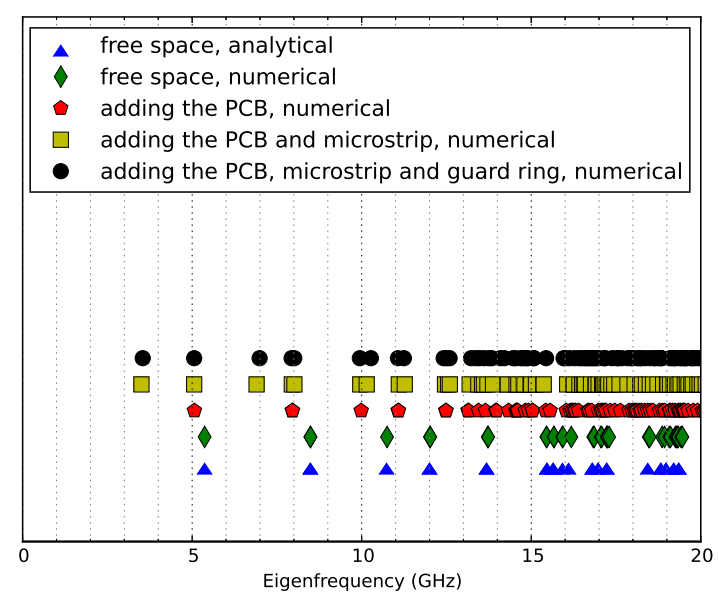

Fig. 11. Resonance frequencies in function of the structural elements. In case of free space, it can be observed that there is a good agreement between the analytical approximation and numerical calculation. Addition of the PCB shifts the resonances down and introduces new resonances in the upper range due to the "cavity" formed by the microstrip, substrate and ground plane.

frequency range. The first phenomena can be explained by Equation (5) where it is shown that the permittivity of the medium is inversely proportional to the frequency. Adding the PCB which has a relative permittivity of 4.6 to a medium with a relative permittivity of 1 increases the "combined" permittivity, thus lowers the resonance frequency.

It can be observed that the microstrip and guard ring introduces new lower frequencies not expected based on the dimensions of the BLS as calculated using Equation (5). It is observed, by introducing near-field point sensors that these lower frequencies are eigenmodes of the "cavity" formed by the microstrip, substrate and ground plane. While the rise of resonances at higher frequencies are related to the microstrip's dimensions.

The degradation of the overall shielding effectiveness due to 
resonances can be observed in Figures 7 and 9. For example, a visually easy to pinpoint degradation of the overall SE occurs at $5.07 \mathrm{GHz}$.

\section{CONCLUSION}

The shielding effectiveness of a board-level shield in function of its bonding was studied by means of full-wave simulations. It was shown that the overall shielding performance improves with increasing number of vias per side for the "classic bonding technique". There is, however, a practical limit to the inter-spacing distance of vias, thus enforcing a limit on the maximum shielding performance achievable with the "classic bonding technique".

It was shown that the overall shielding performance can be improved by applying the "waveguide-below-cutoff" bonding technique, even if these waveguides are formed by parallel rows of vias.

It was also shown by analytical and numerical calculations that the degradation of the overall shielding performance in quite narrow bands around specific frequencies can be related to resonances that are related not only to the dimensions of the BLS, but also to what is present inside the BLS.

Future work: measurements on physical testcases with stripline set-up or reverb set-up [10], [11].

\section{REFERENCES}

[1] K. Armstrong, EMC for printed circuit boards : basic and advanced design \& layout techniques. Nutwood UK, December 2010. Available: www.emcacademy.org/books.asp.

[2] D. Pissoort, M. Mechaik, H. Zeng, C. Shu, C. Jackson, and J. Van Hese, "Influence of the interaction between antenna currents and return currents on the coupling between digital interfaces and on-board antennas," in Electromagnetic Compatibility (EMC), 2013 IEEE International Symposium on, Aug 2013, pp. 1-6.

[3] D. Pissoort, M. Mechaik, H. Zeng, C. Shu, C. Jackson, and J. V. Hese, "Performance degradation due to coupling between high-speed traces and on-board antennas," in Electromagnetic Compatibility (EMC EUROPE), 2013 International Symposium on, Sept 2013, pp. 282-287.

[4] E. Jordan, Electromagnetic waves and radiating systems. Englewood Cliffs, N.J: Prentice-Hall, 1968

[5] H. Ott, Electromagnetic compatibility engineering. Hoboken, N.J: John Wiley \& Sons, 2009.

[6] X. Tong, Advanced materials and design for electromagnetic interference shielding. Boca Raton: CRC Press, 2009.

[7] K. Technologies. EMPro 3D EM Simulation Software. [Online]. Available: http://www.keysight.com

[8] F. Vanhee, B. Vanhee, J. Catrysse, H. Yuhui, and A. Marvin, "Proposed methods to measure the shielding performance of pcb level enclosures," 2009.

[9] H. Yuhui and A. Marvin, "An investigation of the shielding performance of pcb-level enclosures using a reverberation chamber," in Electromagnetic Compatibility, 2007. EMC 2007. IEEE International Symposium on, July 2007, pp. 1-6.

[10] A. Marvin and Y. Cui, "Shielding measurements of equipment enclosures in the radiating near field," Electromagnetic Compatibility, IEEE Transactions on, vol. 49, no. 4, pp. 860-867, Nov 2007.

[11] J. Catrysse, F. Vanhee, D. Pissoort, C. Brull, and G. Vandenbossch, "Towards a global approach for the characterization of ics and on board shielding components," in EMC Europe 2010 : 9th International Symposium on EMC joint with 20th International Wroclaw Symposium on EMC. Wroc?aw: Oficyna Wydawnicza Politechniki Wroc?awskiej, 2010, pp. 889-894.

[12] D. Pissoort, B. Boesman, T. Claeys, J. Pitteman, and J. Catrysse, "Correlating the high-frequency shielding performance of 'on-board' gaskets when characterized using a stripline or reverberation room method," in Electromagnetic Compatibility (EMC), 2014 IEEE International Symposium on, Aug 2014, pp. 128-133.
[13] M. Robinson, T. Benson, C. Christopoulos, J. Dawson, M. Ganley, A. Marvin, S. Porter, and D. Thomas, "Analytical formulation for the shielding effectiveness of enclosures with apertures," Electromagnetic Compatibility, IEEE Transactions on, vol. 40, no. 3, pp. 240-248, Aug 1998.

[14] C. Balanis, Advanced engineering electromagnetics. Hoboken, N.J: John Wiley \& Sons, 2012. 